\title{
Definiendo "en positivo" los neologismos formales: Hacia un análisis cuantitativo de la correlación entre sus características
}

\author{
Miguel SÁNCHez IBÁÑEZ \\ Departamento de Lingüística Aplicada a la Ciencia y a la Tecnología \\ ETSIT de Telecomunicación \\ Universidad Politécnica de Madrid \\ Avenida Complutense, 30 \\ 28040, Madrid \\ E-mail: miguel.sanchezi@upm.es
}

\section{DEFINIENDO "EN POSITIVO" LOS NEOLOGISMOS FORMALES: HACIA UN ANÁLISIS CUANTITATIVO DE LA CORRELACIÓN ENTRE SUS CARAC- TERÍSTICAS}

RESUMEN: En este trabajo planteamos una vía para definir la neología formal desde una nueva perspectiva $\mathrm{y}$, al mismo tiempo, interpretar la correlación entre los que consideramos como los principales parámetros caracterizadores de los neologismos formales en castellano: la consistencia formal, la motivación y la diacronía. Para ello, nos basamos en 3209 neologismos aparecidos en la prensa escrita de Castilla y León (España) entre 2009 y 2016. Aplicamos un baremo propio que favorece a los neologismos que se ajustan a esos parámetros y penaliza a los que no lo hacen y nos servimos de él para analizar las posibles pautas de interdependencia entre los parámetros mencionados. Los resultados muestran que, para nuestro corpus y de acuerdo con la metodología que planteamos, existe una relación de correlación directa entre esos tres parámetros, y que la dimensión diacrónica condiciona de manera significativa la motivación y la consistencia formal.

PALABRAS ClAVES: neologismos; consistencia formal; variación; motivación; diacronía; lexicografia.

SUMARIO: 1. Introducción. 2. Punto de partida y marco de trabajo. 3. Metodología: Definición de parámetros y aplicación del baremo. 4. Metodologia: Análisis de la correlación entre parámetros. 5. Resultados y discusión. 6 . Conclusiones.
DEFINING FORMAL NEOLOGISMS "POSITIVELY": TOWARDS A QUANTITATIVE ANALYSIS OF THE CORRELATION AMONG THEIR CHARACTERISTICS

ABSTRACT: This paper suggests a way to define Spanish formal neologisms through the quantitative analysis of the correlation among their main characteristics: formal consistency, motivation and diachrony. For that purpose, we have classified 3209 neologisms extracted from a group of newspapers published in the region of Castile and Leon (Spain) between 2009 and 2016. We have applied our self-developed scoring scale, which positively assesses the neologisms that conform to those parameters and penalizes those that do not do so. The results show that, for our corpus and using our methodology, there is a direct correlation between the three parameters analysed. We have also proved that diachrony significantly conditions motivation and formal consistency.

KEY WORDS: neologisms; formal consistency; variation; motivation; diachrony; lexicography.

SUMMARY: 1 . Introduction. 2. Background and framework. 3. Methodology: parameters' definition and score scale application. 4. Methodology: analysis of the correlation among parameters. 5. Results and discussion. 6. Conclusions.
UNE DEFINITION "EN POSITIF" DES NEOLOGISMES FORMELS : VERS UNE ANALYSE DE LA CORRELATION ENTRE SES CARACTERISTIQUES

RÉSUMÉ: Cet article propose une méthodologie pour définir la néologie formelle à partir d'une nouvelle perspective, qui permet en même temps d'interpréter la corrélation entre les paramètres que l'on considère les plus pertinents pour caractériser les néologismes formels en espagnol : la consistance formelle, la motivation et la diachronie. On a classifié 3209 néologismes apparus dans plusieurs journaux publiés dans la région de Castille-et-Léon (Espagne) entre 2009 et 2016 , et on a appliqué notre propre barème de notation, qui favorise les néologismes concordants avec nos paramètres tout en pénalisant ceux qui ne concordent pas. Les résultats montrent que, pour notre corpus et conformément à notre méthodologie, il y a une corrélation directe entre ces trois paramètres, et que la diachronie conditionne significativement la motivation et la consistance formelle dans la création de nouveaux mots en espagnol.

MOTS CLÉS: néologismes; consistance formelle; variation; motivation; diachronie; lexicographie.

SOMMAIRE: 11 . Introduction. 2. Point de départ et cadre du travail. 3. Méthodologie: définition des paramètres et application du barème de notation. 4 . Méthodologie: analyse de la corrélation entre les paramètres. 5 . Résultats et discussion. 6 . Conclusions. $\begin{array}{ll}\text { Fecha de Recepción } & 11 / 12 / 2017 \\ \text { Fecha de Revisión } & 17 / 07 / 2018 \\ \text { Fecha de Aceptación } & 31 / 07 / 2018 \\ \text { Fecha de Publicación } & 01 / 12 / 2018\end{array}$ 


\section{Definiendo "en positivo" los neologismos formales: Hacia un análisis cuantitativo de la correlación entre sus características}

Miguel SÁNCHEZ IBÁÑEZ

\section{INTRODUCCIÓN}

Tradicionalmente, se ha considerado que el término neología servía para designar, por un lado, a la disciplina que estudiaba a los neologismos y, por el otro, al campo de trabajo aplicado de esta (Cabré, 2016: 20). Sin embargo, los trabajos prácticos, destinados principalmente a la detección y clasificación de las unidades de nuevo cuño y siempre ligados a otro tipo de proyectos lingüísticos o traductológicos, han acaparado el significado de dicha palabra, arrinconando reflexión teórica y convirtiéndola en eterna subsidiaria de las conclusiones de los trabajos aplicados. No obstante, parece lógico pensar que detrás de todos ellos siempre existe una mínima fundamentación teórica que sirve de andamio para sustentar los análisis concretos.

Quizá por ese arrinconamiento, la definición de neologismo ha tendido a formularse en negativo, basada en criterios de exclusión y de eliminación (Guilbert, 1973; Rey, 1976; Cabré, 1999, Hormigo Díaz, 2007), ya que a la luz de las taxonomías y las clasificaciones que se han sucedido a lo largo de las últimas décadas (Sablayrolles, 2000) podriamos concluir que ha sido más sencillo decir qué no era una unidad de nuevo cuño que lo contrario. Sabemos que se trata de unidades que no son estables desde un punto de vista formal y semántico, que no tienen por qué responder a necesidades denominativas puramente denotativas, que pueden confundirse con rarezas y ocurrencias léxicas (Vega Moreno, 2015) y que tienden, en definitiva, a situarse en las periferias de las lenguas. Pero la relatividad en la que siempre descansa la naturaleza neológica de las unidades léxicas (Cabré, 2015) limita que se puedan fijar sus características en positivo, de una manera objetiva y sistemática. Con el fin de esbozar algunas premisas que permitan cambiar ese paradigma, este artículo se centra en caracterizar un grupo de neologismos formales, y en plantear una estrategia para calibrar la correlación existente entre tres parámetros que consideramos determinantes en dicha caracterización: la consistencia formal, la motivación y la diacronía.

En primer lugar, explicamos cómo hemos diseñado un baremo de puntuación que nos ha ayudado a ordenar un grupo de 3209 neologismos función de su grado de adecuación a los ya mencionados parámetros. En segundo lugar, detallamos la manera en que hemos intentado definir de manera cuantitativa la correlación existente entre ellos, para intentar huir, de alguna manera, de la ya mencionada relatividad. Nuestra hipótesis de partida, es, en consecuencia, que el análisis de la correlación entre la consistencia formal, la motivación y los aspectos cronológicos de las unidades de nuestro estudio da claves pertinentes para definir los neologismos formales 
de manera objetiva, englobadora y en positivo. Por otra parte, también pretendemos determinar, en primer lugar, si existe un parámetro que condicione a los otros, y en segundo lugar, averiguar si la correlación que se establece entre ellos tiende a ser directa (cuando uno aumenta, los demás también lo hacen) o inversa (cuando uno aumenta, los demás disminuyen).

\section{PUNTO DE PARTIDA Y MARCO DE TRABAJO}

Nuestro análisis se basa en un conjunto de neologismos clasificados por el Observatorio de Neología de la Universidad de Salamanca (NeoUSAL), que estudia la neología de la región española de Castilla y León y es miembro de la red NEOROC de Observatorios de Neologia, coordinada desde el Observatorio de Neologia de la Universitat Pompeu Fabra (OBNEO). Esos neologismos fueron extraídos de manera semiautomática de un corpus compuesto por dos números mensuales escogidos de manera aleatoria de diez periódicos publicados en Castilla y León ${ }^{1}$ entre los años 2009 y 2016, como recoge el Gráfico 1. En total, recogimos muestras de 3209 neologismos formales diferentes.

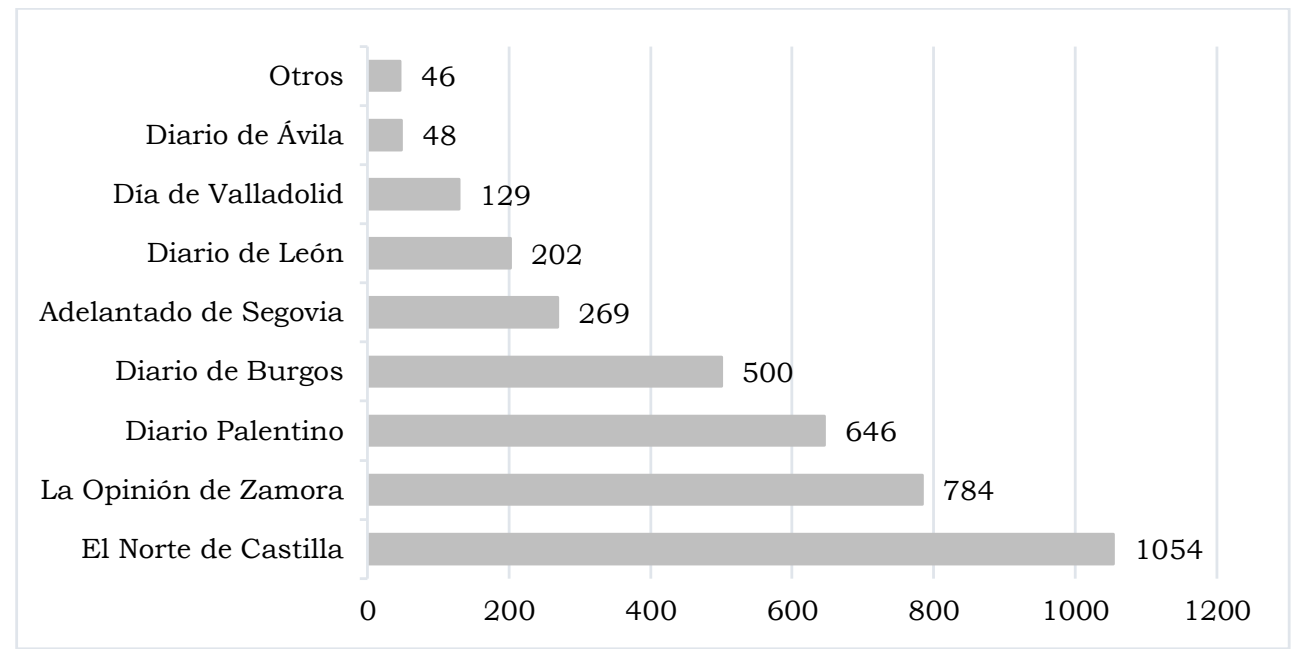

Gráfico 1: Número de neologismos extraídos de cada periódico

La extracción de las unidades siguió la metodologia y directrices marcadas desde OBNEO, comunes a todos los observatorios de la red (OBNEO, 2004: 3-29). Dicha metodología descansa sobre el criterio de exclusión lexicográfica para hacer la selección automática de candidatos a neologismos 2 .

${ }^{1}$ El Norte de Castilla, El Día de Valladolid, El Diario Palentino, Diario de León. Diario de Burgos, Adelantado de Segovia, La Opinión de Zamora, La Gaceta de Salamanca, Diario de Ávila, El Mundo (Edición Castilla y León).

${ }^{2}$ Cabré y Estopá (2009: 20) definen este criterio como aquel que permite 
A pesar de su utilidad en una primera criba, pronto nos dimos cuenta de que, tal y como afirman Freixa y Solé (2006: 137) ese criterio permitía que unidades no neológicas aparecieran también en la lista de posibles neologismos, como sucedia con los gentilicios (terracampino -na, fermosellano, na...), las unidades formadas a partir de elementos extremadamente previsibles (por ejemplo, las formadas con el prefijo ex -, como exconsejero, -ra o excandidato -ta) o aquellas que en su momento fueron neológicas pero que cayeron en desuso antes de verse incorporadas a los diccionarios (pastorella, escultopintura...).

Con el fin de refinar esa búsqueda, diseñamos una segunda criba sistemática, objetiva y coherente con la primera: un baremo que puntuara positivamente a las unidades de una naturaleza neológica más inequivoca. Para decidir cómo diseñar ese baremo, primero establecimos las características neológicas que queriamos que evaluara:

- Variación y consistencia formal: dado que íbamos a trabajar con unidades formalmente inestables, que en muchos casos habian sido importadas de otros códigos, nos parecía relevante poder calibrar hasta qué punto una unidad presentaba variantes formales que denotaran un grado determinado de consistencia o variación en ese sentido.

- Uso: probar la utilización de las unidades por parte de los hablantes también fue un criterio relevante para nosotros, puesto que entendimos que, en la medida en que pudiéramos dar con evidencias de este aspecto, podriamos justificar la pertinencia de su existencia.

- Claridad semántica para los usuarios: creímos relevante tener en cuenta la aparición de los neologismos en contextos en los que se explicara su significado, o se añadieran sinónimos o glosas. Nos pareció que el hecho de que los usuarios de un neologismo creyeran oportuno añadir ese tipo de añadidos ponía de manifiesto la percepción de esa unidad como dificil de comprender y, en definitiva, anómala en cierto sentido.

- Cronología, implantación y actualidad: pensamos que determinar la aparición o ausencia de los neologismos y su trayectoria a lo largo de un periodo determinado de tiempo en uno o varios corpus podía ser útil. Del mismo modo, y dado que trabajábamos con neologismos aparecidos en

considerar neologismo cualquier palabra que no aparece en un corpus lexicográfico de exclusión. En nuestro caso, los diccionarios escogidos para la criba fueron Diccionario de la Lengua Española, (Real Academia Española, 2014) y el LEMA: Diccionario de la Lengua Española (Battaner et al., 2001). Las ventajas del vaciado automático frente al vaciado manual son muy relevantes, ya que, como dicen Freixa y Solé (2006: 146) el 75 \% de los neologismos de lengua general son mayoritariamente neologismos léxicos formales monolexemáticos, es decir, aquellos que esta estrategia de selección detecta con mayor éxito. 
textos periodísticos y, en consecuencia, presumiblemente ligados a la actualidad, también consideramos que este hecho debía estar presente en nuestro baremo.

En consecuencia, decidimos que nuestro baremo puntuara positivamente las unidades que cumplieran estos requisitos y las penalizara en caso contrario.

\section{METOdología: DEFINICIÓN DE PARÁMETROS Y APLICACIÓN DEL BAREMO}

\subsection{CONSISTENCIA FORMAL}

El estudio de unidades aparecidas en medio escrito propició que nos centráramos en sus características formales y la manera en que estas se representaban gráficamente. Nos parecía interesante baremar esta característica de una manera exhaustiva, pues se encuentra en la raíz de la caracterización de muchos neologismos, dada la inestabilidad que suelen manifestar en el plano formal como parte de su proceso de estabilización y adecuación al código (Loubier, 2011). Sánchez Manzanares, (2013: 118) recuerda, a propósito de los préstamos neológicos, que son una de las categorías más nutridas de unidades que hemos baremado, que "suelen alternar con la forma original durante un tiempo, hasta que se asienta en el uso una de las dos formas". En nuestro caso, y tal y como muestra la primera tabla del Anexo 1 , hemos favorecido a las unidades con mayor frecuencia absoluta y menor número de variantes formales.

\subsection{Motivación}

En este caso, pretendimos evaluar de manera sistemática y cuantitativa en qué medida existen razones que justifiquen y refuercen la existencia de los neologismos de nuestro estudio.

Casado Velarde (2015: 22) dice que "la aparición de un neologismo se encuentra en la necesidad de denominar una nueva realidad (...) es una necesidad notativa o referencial", pero también hace referencia a la necesidad "expresiva" influida por la moda, la cultura o la mentalidad de los hablantes, haciendo alusión a la tradicional división entre neología denominativa y estilística. Podriamos decir que la principal razón que subyace a la existencia de una unidad léxica es la de su utilidad para denominar un concepto determinado, aunque pueda considerarse más o menos necesaria. De nuevo centrándose en los préstamos neológicos, Sánchez Manzanares (2013: 123) señala que su uso "puede responder tanto a una necesidad denominativa como a una estrategia comunicativa, por lo que no debe subordinarse su sanción exclusivamente a la variable de necesidad denominativa”. Esa dilogia entre necesidad y estrategia ya la trató Guilbert (1975) al distinguir entre dos motivaciones para acoger una nueva unidad en el seno 
de un código establecido: las lingüísticas, como la necesidad de rellenar un vacío léxico en el código importador, o las extralingüísticas, como la tendencia de una comunidad a querer parecerse a otra mediante su código. Asimismo, dicha dilogía se ha ido articulando en lo que ya hemos apuntado: la neologia estilística, que es prescindible y accesoria, y la dilogía denominativa, que es necesaria y cumple con una función referencial (Guilbert, ibid.; Sablayrolles, 2000; Sablayrolles y Thoiron, 2003). Su análisis resulta ser especialmente relevante porque en muchos casos las unidades neológicas cuentan con otras unidades o estrategias denominativas equivalentes, lo que hace que estas puedan considerarse, a priori, un recurso más estilístico que referencial. Si a esto añadimos el factor de aceptabilidad y adecuación a la norma, podríamos concluir con facilidad que un neologismo carece de utilidad si cae en la redundancia denominativa, aunque existan motivos "estilisticos" que hayan propiciado su aparición.

No obstante, esta doble clasificación ha recibido numerosas objeciones, por lo ficticio que resulta el carácter excluyente de ambas categorias y lo inconsistente que puede llegar a ser su aplicación. Por ejemplo, algunos autores, como Estornell (2009) o Loubier (2011) relacionan los neologismos estilísticos con una implantación escasa, algo que entra en clara contradicción con muchos de los ejemplos de nuestro estudio, donde abundan las unidades de uso repetido y consistente, como fracking, app o coworking, para las que existen equivalentes en castellano recomendados por diversos organismos reguladores ${ }^{3}$. A este respecto, Sablayrolles (2016: 46) afirma que "il y a toujours opération de nomination, avec réglage du sens, d'un point de vue praxématique. De ce point de vue, rien n'est jamais sans raison (...) les néologismes ont toujours une ou des raisons d'être."

Con el fin de superar esa división, Llopart-Saumell (2016) propuso una serie de parámetros de análisis que ayudaran a determinar qué tipo de motivación subyacía a un conjunto de neologismos recogido siguiendo la misma metodología de nuestro estudio: neologismos formales detectados en prensa escrita mediante el criterio lexicográfico de exclusión. Los parámetros de análisis propuestos por esta autora fueron: conocimiento del mundo, uso, estabilidad, novedad, posición discursiva y contexto discursivo. Para organizar esta parte de nuestro baremo, y tomando como base los parámetros expuestos por Llopart-Saumell, hemos decidido favorecer a las unidades presentes en corpus ajenos a nuestro estudio ${ }^{4} \mathrm{y}$ a aquellas que aparecen

3 La Fundación del Español Urgente, organismo asesorado por la Real Academia Española, (www.fundeu.es) recomienda, en estos tres ejemplos: fractura hidráulica o hidrofacturación, aplicación y cotrabajo, respectivamente, y desaconseja el uso de los préstamos, a pesar del creciente uso, implantación y consistencia de las mismas, puestos de manifiesto nuestro estudio.

4 En concreto, el Corpus de Referencia del Español Actual (CREA) (http://corpus.rae.es/creanet.html) y el Corpus de la Red de observatorios de neología del español (BOBNEO)(http://obneo.iula.upf.edu/bobneo/index.php). 
provistas de marcadores metadiscursivos adyacentes, tal y como muestra la segunda tabla del Anexo 1.

\subsection{DiACRONÍA}

Consideramos importante dedicar una categoría al elemento cronológico porque está en la propia base del concepto de neologismo y, como tal, se debe tener en cuenta a la hora de caracterizar a estas unidades.

Toute autre référence au temps qui serait de nature linéaire se heurte à l'impossible consensus quand vient le moment de définir cette durée en termes de jours, de mois ou d'années, le chiffre déterminé devant s'appliquer à tous les néologismes. L'évaluation repose sur des conditions très variées, dont plusieurs sont de nature idiosyncrasique et d'origine extralinguistique.

Boulanger, 2010: 69

Para baremar la diacronía, tuvimos en cuenta tres conceptos: novedad, implantación y actualidad, que se encuentran resumidos en la tercera tabla del Anexo 1 y que definimos de la siguiente manera:

Novedad: a propósito de este concepto, Boulanger (ibid.: 63) afirma que es

le premier stade du néologisme, état qui freine souvent l'emploi du mot ou qui instaure et/ou entretient l'instabilité, l'insécurité, la méfiance (...) Pendant un temps plus ou moins long, le signifiant est senti comme un corps étranger".

Creímos necesario partir de esa noción a la hora de decidir cómo puntuar a nuestros neologismos, ya que la combinación de una puntuación alta en este parámetro (ligado, como dice Boulanger, a la inestabilidad y a la desconfianza), unida a otra puntuación elevada en unidades de uso extendido, a las que hemos favorecido en el apartado de motivación comprobando su presencia en CREA y en BOBNEO, favorecería a las unidades recientes, pero, al mismo tiempo, muy presentes en la lengua. En consecuencia, decidimos dar una mayor puntuación a aquellos neologismos de aparición más reciente en BOBNEO e ir disminuyéndola a medida que esta se alejaba en el tiempo, hasta el punto de puntuar de manera negativa a los aparecidos en los primeros años del Observatorio, especialmente a los que constaban desde 1989, fecha en la que se inició la recopilación de unidades.

Implantación: a este respecto, partimos de la definición de Quirion (2003: 13-14), que entiende la implantación léxica como la gestión de datos de ese tipo orientada a su difusión (proceso), y también como el uso posterior de dicho vocabulario (resultado). Para nuestro estudio nos fue útil la primera acepción, ya que pretendimos evaluar la progresión, ya fuera ascendente o descendente, de la frecuencia absoluta de apariciones de nuestros neologismos en el lapso de tiempo que cubría nuestro corpus, es decir, de 2009 a 2016. En un principio, planteamos medir esa progresión año a año, pero dada la baja frecuencia de la mayoría de unidades (solo un 18,6\% de ellas 
aparecian más de una vez) optamos por dividir el lapso de tiempo en dos mitades y favorecer a aquellas unidades que aparecían más en el tramo más reciente, 2013-2016 (mostraban una implantación ascendente) y penalizar a las que aparecian más en el tramo más antiguo, 2009-2012. Como es obvio, la implantación solo se pudo baremar con aquellos neologismos que aparecían un mínimo de dos veces en nuestro corpus.

Actualidad: Entendiamos que los neologismos, y más si aparecían en prensa escrita, como era nuestro caso, ganarian en relevancia en la medida en que denominaran conceptos actuales, puede que todavía en fase de configuración o de una estabilidad incierta y una perdurabilidad no garantizada. En consecuencia, teniendo en cuenta este elemento también favorecíamos en nuestro baremo aquellos neologismos que no tenían un alto grado de consistencia formal o cuyo uso no estaba probado más allá de nuestro corpus pero que, en contrapartida, eran los más novedosos. Por tanto, decidimos puntuar positivamente y de manera específica, a aquellos neologismos que únicamente estuvieran datados en nuestro corpus en 2016.

En los tres casos planteamos un baremo basado en contrastes, ya fuera entre las propias unidades del estudio, entre ellas y otras aparecidas en otros corpus, o entre distintas características que estas pudieran presentar. El Anexo 2 muestra las 15 unidades con mayor puntuación, de las 3209 analizadas, tras la aplicación del baremo.

\section{METOdOLOGÍA: ANÁliSiS dE LA CORRELACIÓN ENTRE PARÁMETROS}

Como vemos en el Anexo 2, hay neologismos que obtuvieron puntuaciones muy descompensadas entre unos parámetros y otros. Esto nos hizo pensar en las relaciones que se establecian entre consistencia formal, motivación y diacronia: ¿existía un parámetro subordinado a los otros dos? ¿Había algún tipo de tendencia o pauta de proporcionalidad en las puntuaciones obtenidas en cada uno de ellos, en función, por ejemplo, del procedimiento de formación de las unidades? Para dar respuesta a estas preguntas, que creíamos que podrían ayudarnos a cumplir con nuestro objetivo de dar con nuevas claves objetivas para definir a los neologismos, decidimos dar un paso más en nuestro análisis y estudiar de manera cuantitativa las relaciones de condicionamiento y subordinación entre nuestros tres parámetros.

\subsection{UMBRAL DE SUPERACIÓN DE PARÁMETROS Y VALIDACIÓN DE LA MUESTRA}

Para proseguir con nuestro estudio, decidimos seleccionar aquellas unidades con un mínimo de +4 puntos en nuestro baremo. Fueron 1695, es decir, un 52,8\% de las 3209 con las que iniciamos el estudio. Por otra parte, también establecimos un umbral de superación para cada uno de los parámetros: fue de $>0$ puntos para consistencia formal y diacronia, y de $\geq+2$ para 
motivación. En la Tabla 1 se observa el número de neologismos que superaron el indicador establecido para cada uno de los parámetros, tanto en el cómputo global como en los 1695:

\begin{tabular}{|c|c|c|c|c|}
\hline \multirow{2}{*}{ Parámetro } & \multicolumn{2}{|c|}{$\begin{array}{c}\text { Neologismos que lo superan del } \\
\text { total (3209) }\end{array}$} & $\begin{array}{c}\text { Neologismos que lo superan de los } \\
\text { que obtuvieron } \geq+4 \text { en el baremo } \\
\text { (1695) }\end{array}$ \\
\cline { 2 - 5 } & $\begin{array}{c}\text { Frecuencia ab- } \\
\text { soluta }\end{array}$ & $\%$ & $\begin{array}{c}\text { Frecuencia abso- } \\
\text { luta }\end{array}$ & $\%$ \\
\hline $\begin{array}{c}\text { Consistencia for- } \\
\text { mal (>0) }\end{array}$ & 792 & $24,6 \%$ & 686 & $40,4 \%$ \\
\hline Motivación (>+2) & 1113 & $34,6 \%$ & 737 & $43,4 \%$ \\
\hline Diacronia (>0) & 2184 & $68 \%$ & 1415 & $83,4 \%$ \\
\hline
\end{tabular}

Tabla 1: Neologismos que superaron cada parámetro

Consideramos que estos datos confirmaban que la muestra de 1695 neologismos con la que íbamos a proseguir el análisis era representativa por dos motivos:

En primer lugar, fue significativa la coincidencia en el orden de los parámetros, de acuerdo con su nivel de superación: tanto si tomábamos el total de neologismos como la muestra seleccionada, el parámetro que mayor porcentaje de superación obtenía era el de diacronía, seguido del de motivación y, por último, el de consistencia formal.

En segundo lugar, la proporción de neologismos que superaban cada uno de los parámetros y se encontraban entre los 1695 con mayor puntuación era significativa, como podemos ver en la Tabla 2: en los tres casos más de la mitad de las unidades que cumplian ese requisito se encontraban entre las 1695 de mayor puntación, y en un caso la proporción se acercaba al 90\%:

\begin{tabular}{|c|c|c|c|}
\hline Parámetro & $\begin{array}{c}\text { Cantidad de unida- } \\
\text { des que lo superan } \\
\text { (del total) }\end{array}$ & $\begin{array}{c}\text { Cantidad de unida- } \\
\text { des con } \geq+4 \text { puntos } \\
\text { que lo superan }\end{array}$ & $\begin{array}{c}\text { Porcentaje de unida- } \\
\text { des que lo superan y } \\
\text { tienen } \geq+4\end{array}$ \\
\hline Consistencia formal & 792 & 686 & $86,6 \%$ \\
\hline Motivación & 1113 & 737 & $66,2 \%$ \\
\hline Diacronía & 2184 & 1415 & $64,7 \%$ \\
\hline
\end{tabular}

Tabla 2: Proporción de neologismos seleccionados que han superado cada parámetro 


\subsection{TIPOS DE CORRELACIÓN ANALIZADOS}

Al hablar de correlaciones nos referimos a las interdependencias establecidas entre los parámetros que hemos baremado, y que, convenientemente analizadas y desglosadas, pueden revelar condicionamientos y vínculos entre ellos. Existen distintas maneras de medir este tipo de relaciones, y partiendo de nociones estadísticas básicas, hemos decidido centrarnos en tres tipos: uno de ellos basado en un elemento estadístico ya existente (coeficiente de correlación de Pearson) y otros dos ideados por nosotros mismos a la luz de nuestro baremo.

\subsubsection{CORRELACIÓN GLOBAL}

Para analizar la manera en que se interrelacionaban los tres parámetros, comenzamos por determinar la proporción de unidades que, habiendo superado uno de los tres parámetros de acuerdo con los umbrales que habíamos establecido, superaban los otros dos, tal y como representa la Figura 1.

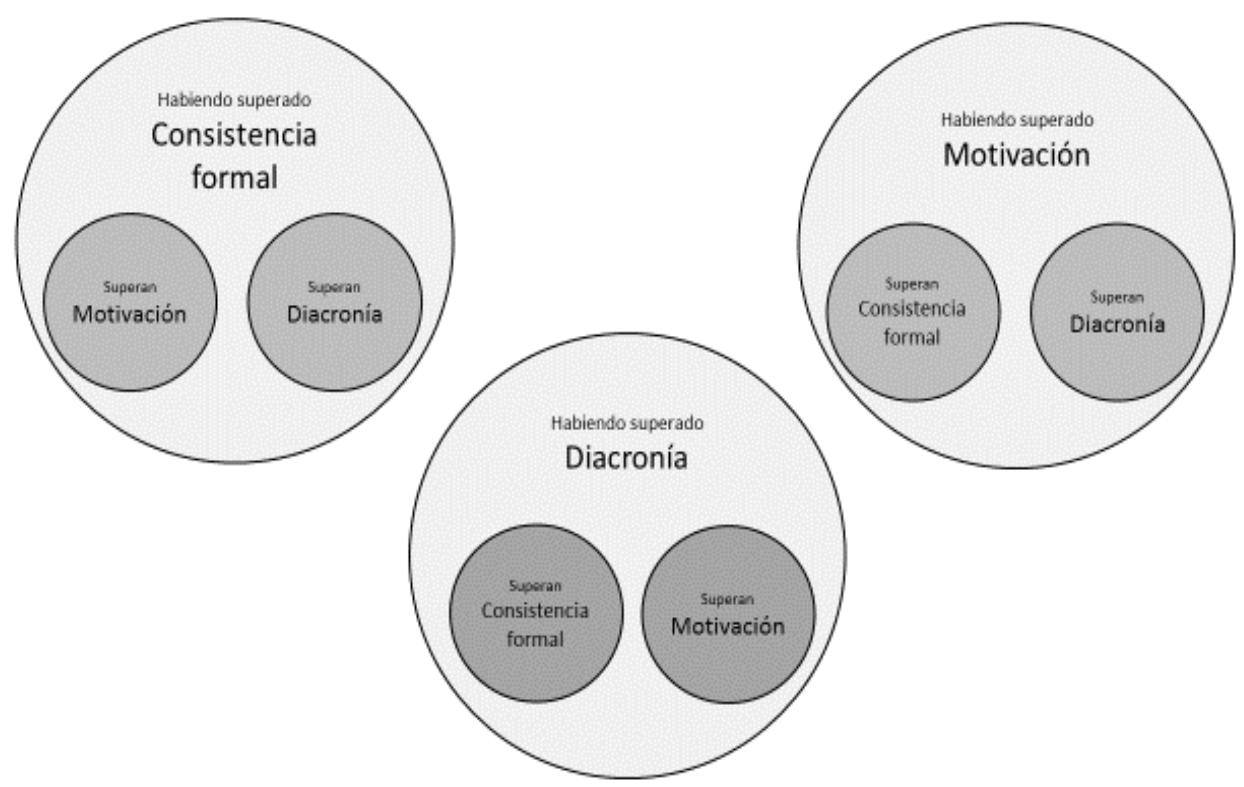

Figura 1: Organización de los parámetros para el análisis de sus correlaciones

La Tabla 3 muestra dicha información, tanto en términos absolutos como en porcentajes. Asimismo, incluye el promedio entre ambos parámetros para cada uno de los tres supuestos contemplados. 


\begin{tabular}{|c|c|c|c|c|c|c|c|c|c|}
\hline \multirow{3}{*}{$\begin{array}{c}\text { TOTAL: } \\
1695\end{array}$} & \multirow{3}{*}{\multicolumn{2}{|c|}{$\begin{array}{l}\text { 2. Superan } \\
\text { también } \rightarrow\end{array}$}} & \multicolumn{2}{|c|}{ Consistencia } & \multicolumn{2}{|c|}{ Motivación } & \multicolumn{2}{|c|}{ Diacronía } & \multirow{3}{*}{$\begin{array}{l}\text { Prome- } \\
\text { dio }\end{array}$} \\
\hline & & & \multicolumn{2}{|c|}{686} & \multicolumn{2}{|c|}{737} & \multicolumn{2}{|c|}{1415} & \\
\hline & & & Frec. & $\%$ & Frec. & $\%$ & Frec. & $\%$ & \\
\hline \multirow{3}{*}{$\begin{array}{l}\text { 1. Ha- } \\
\text { biendo su- } \\
\text { perado } \rightarrow\end{array}$} & $\begin{array}{c}\text { Consist. } \\
\text { formal }\end{array}$ & 686 & & & 384 & $52,1 \%$ & 430 & $30,3 \%$ & $41,2 \%$ \\
\hline & $\begin{array}{l}\text { Motiva- } \\
\text { ción }\end{array}$ & 737 & 384 & $55,9 \%$ & & & 480 & $33,9 \%$ & $44,9 \%$ \\
\hline & $\begin{array}{c}\text { Diacro- } \\
\text { nía }\end{array}$ & 1415 & 430 & $62,6 \%$ & 480 & $65,1 \%$ & & & $63,85 \%$ \\
\hline
\end{tabular}

Tabla 3: Proporción de neologismos que, habiendo superado un parámetro, superan los otros dos

De los datos reflejados en esta tabla podemos concluir que la proporción de superación de dos parámetros siempre y cuando se hubiera superado el tercero era muy similar en los casos de la consistencia formal y la motivación, pero sensiblemente superior en el de la diacronía, que rebasa el $60 \%$ de promedio.

En términos globales, estos datos nos hacen pensar que el carácter novedoso de una unidad, su remisión a una realidad actual o su implantación ascendente en nuestro corpus (elementos que consideramos al baremar la diacronia) no tenían por qué implicar una excesiva consistencia formal ni motivación. Del mismo modo, también comprobamos que las posibilidades de que un neologismo ganara en relevancia (de acuerdo con nuestro baremo) sin tener en cuenta su novedad aumentaban si tomábamos su motivación y su consistencia formal de manera conjunta, y no por separado.

No obstante, esa conclusión tan solo constataba algo esperable: el uso de una unidad durante un corto lapso de tiempo reciente le imprime actualidad pero, al mismo tiempo, le hace perder en consistencia. Comprobamos, en definitiva, que el carácter novedoso y la implantación ascendente de un neologismo en un periodo de tiempo reciente tendian a diferir de su consistencia formal y del grado de motivación de su uso. Se hacía necesario, por tanto, seguir profundizando en esa correlación para dar con alguna clave que no fuera tan obvia.

\subsubsection{CORRELACIÓN GLOBAL SEGÚN PROCEDIMIENTO DE FORMACIÓN NEOLÓGICA}

Los datos que hemos presentado en la Tabla 3 nos indican la proporción de neologismos que superaron un parámetro habiendo superado los otros dos, pero de un modo global. Con el fin de profundizar en el análisis y comprobar si esas proporciones se mantenían constantes en todos los tipos de 
neologismos según su procedimiento de formación, desglosamos los datos atendiendo a los procedimientos de formación neológica de las unidades estudiadas, como recoge el Gráfico 2, y que explicamos a continuación:

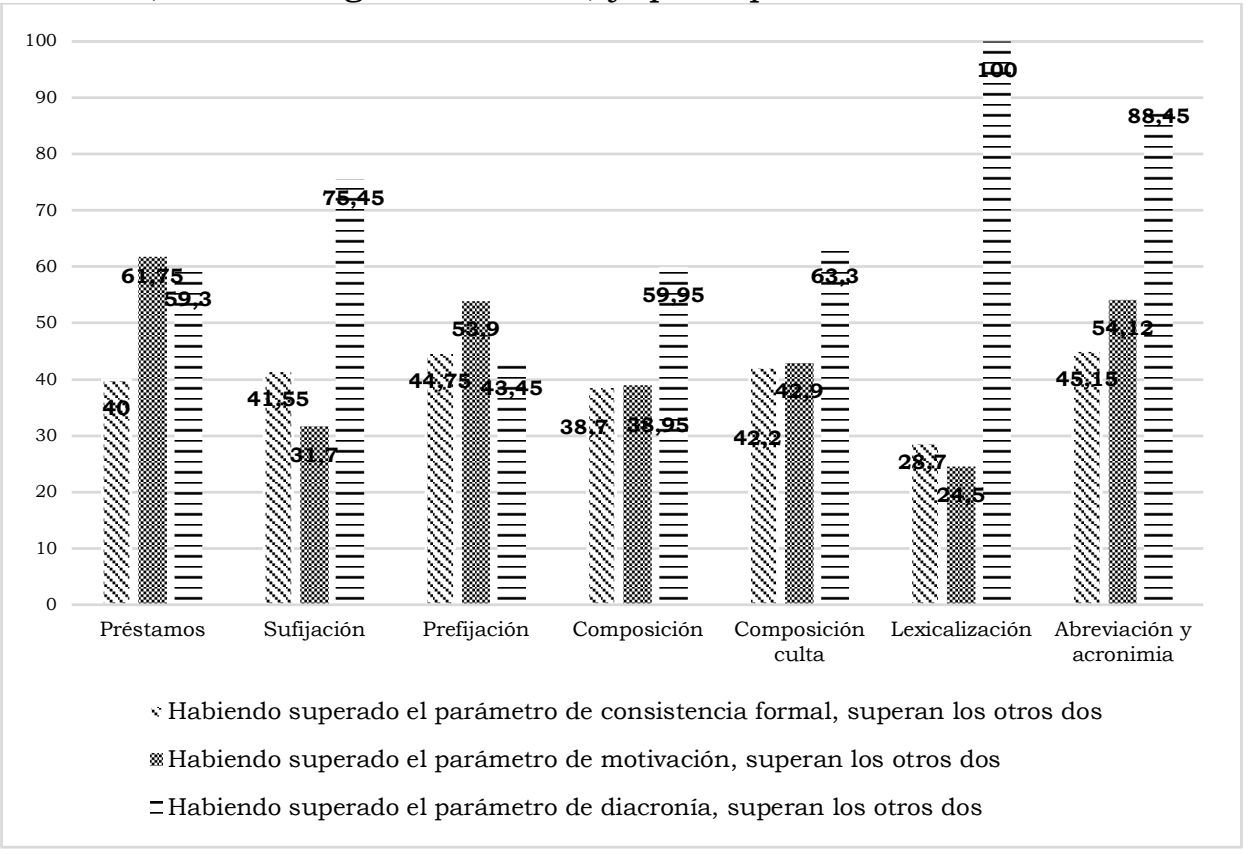

Gráfico 2: Porcentajes de neologismos que, habiendo superado un parámetro, superan los otros dos, en función de su procedimiento de formación

\section{Préstamos neológicos}

Entendemos por préstamo neológico aquella unidad cuya novedad está marcada por su reciente importación de otro código lingüístico, como like, coworking o selfie. Observamos un equilibrio bastante compensado entre los tres casos, si bien la consistencia formal se encuentra por debajo de los otros dos parámetros, algo lógico si tenemos en cuenta que estamos hablando de unidades importadas de otros códigos que tienden a presentar múltiples tipos de variación formal. De hecho, por razones de espacio no aparece en el gráfico, pero es pertinente reseñar el porcentaje de préstamos que, siendo formalmente consistentes también superaron el parámetro de diacronía fue solo del 30,4\%, lo que confirma lo esperable: cuanto más reciente es un préstamo, más variación formal presenta, dado que se encuentra en las primeras etapas de asimilación a la lengua receptora.

\section{Sufijación}

En este caso, la neología surge de la adición novedosa de un sufijo a un radical ya existente, como en el caso de troquelador, alertante o gadafista. 
Para definir este tipo de neologismos, la diacronía es determinante. Por el contrario, la motivación es un parámetro caracterizador débil, algo que podría deberse a la transparencia semántica que suelen presentar estos neologismos, lo que reduce sus posibilidades de presentar marcadores metadiscursivos que aclaren su significado y, en consecuencia, los hace obtener una menor puntuación en ese parámetro.

\section{Prefijación}

La neología por prefijación consiste en la adición de un prefijo a un radical ya existente, como en los casos de desprivatizar, prenavideño -ña o copago. Es llamativo el hecho de que, al contrario de lo que sucedía con los neologismos formados por sufijación, en este caso la motivación es el parámetro más determinante.

\section{Composición}

En este caso nos referimos a aquellas unidades novedosas formadas por dos radicales, ya sean simples o complejos, como sociosanitario-ria, bombalapa o globoflexia. El principal factor caracterizador en este caso es el diacrónico, con la consistencia formal y la motivación prácticamente empatados. Aunque el gráfico no lo recoge, es importante señalar que sólo un $20 \%$ de unidades superaban el factor diacrónico, habiendo superado también el parámetro de consistencia formal, lo que nos coloca en un escenario similar al de los préstamos: a mayor novedad del neologismo compuesto, mayor variación formal.

\section{Composición culta}

Este procedimiento de formación es similar al anterior, con la salvedad de que al menos uno de los dos radicales ha de ser de origen grecolatino, como sucede en micromecenzago, enoturismo o geolocalización. El peso de la diacronía es evidente, algo a lo que se une una correlación muy estrecha entre consistencia formal y motivación. De nuevo, el modesto porcentaje de unidades que, habiendo superado el parámetro de consistencia formal, no supera el de la diacronía (29\%) relativiza la supuesta estabilidad formal que se presupone a este tipo de neologismos.

\section{Lexicalización}

Este tipo de neologismos surgen cuando una forma flexiva de un paradigma, ya sea verbal o nominal, cobra entidad propia como unidad con significado propio, como en el caso de centimazo, reasfaltado o altarcillo. El factor diacrónico ha sido absolutamente determinante para la caracteriza- 
ción de este tipo de neologismos, dado que todos ellos superan ese parámetro. En otras palabras: lo que hace que los neologismos por lexicalización detectados en nuestro estudio sean relevantes es, simplemente, su novedad.

\section{Abreviación o acronimia}

Unidades formadas por el acortamiento de otra unidad ya existente o por la unión de los acortamientos de varias unidades, como en el caso de ceas, nini, o electrolinera. De nuevo, se repite la misma pauta que en otros neologismos analizados: el elemento diacrónico es determinante y la consistencia formal es el parámetro más débil.

\subsubsection{Coeficiente de CORRelación de Pearson}

A continuación, decidimos introducir en nuestro análisis un elemento estadístico que nos ayudara a ver con mayor claridad las relaciones que establecidas entre los tres parámetros: el coeficiente de correlación de Pearson, que permite medir el grado de relación entre varias variables aleatorias cuantitativas. Su resultado siempre es una cifra comprendida entre $-1 \mathrm{y}+1$. Un índice de correlación de -1 refleja una relación totalmente inversa entre las variables, es decir, que cuando una de ellas aumenta, la otra disminuye en la misma medida. Por el contrario, si el índice es de +1 , la relación sería directa, es decir, cuando una variable aumenta, la otra hace lo propio, en la misma medida.

La Tabla 4 muestra los índices de correlación existentes entre nuestras tres variables, que son nuestros parámetros. La correlación es directa en los tres casos, pero sensiblemente menor si contrastamos la diacronía y la consistencia formal, lo que confirma la divergencia que hemos venido señalando.

\begin{tabular}{|c|c|c|c|}
\hline & $\begin{array}{c}\text { Consistencia } \\
\text { formal }\end{array}$ & Motivación & Diacronia \\
\hline Consistencia formal & 1 & 0,94534137 & 0,79907683 \\
\hline Motivación & 0,94534137 & 1 & 0,94525031 \\
\hline Diacronia & 0,79907683 & 0,94525031 & 1 \\
\hline
\end{tabular}

Tabla 4: Coeficiente de correlación de Pearson entre los elementos baremados

\subsubsection{CORRELACIÓN PROGRESIVA}

Al determinar la correlación global entre nuestros tres parámetros hemos pasado por alto la posición de las unidades en la clasificación que hemos obtenido tras aplicar nuestro baremo. Creímos que resultaba poco fiel a la realidad considerar similares dos unidades entre las que pueden mediar 
hasta catorce puntos de diferencia, como sucede entre fracking, la primera de nuestra clasificación, que tiene 18 puntos, y cualquiera de las que hayan obtenido 4. Por ese motivo, decidimos analizar de nuevo la correlación, pero teniendo en cuenta ese nuevo elemento, en lo que hemos denominado como correlación progresiva. Para ello, seguimos los siguientes pasos:

En primer lugar, comprobamos el número de unidades que superaban cada parámetro por separado, pero en lugar de hacerlo en términos absolutos, lo comprobamos dividiendo en análisis en tramos progresivos de la clasificación: primero, atendiendo a los doscientos neologismos con mayor puntuación, después teniendo en cuenta los cuatrocientos primeros, y así sucesivamente, añadiendo doscientos a cada cómputo, hasta llegar a los 1695 totales. La dirección de las líneas de cada parámetro (Gráfico 3) nos dio las primeras claves: vimos que la proporción de unidades que superaban el parámetro de consistencia formal iba decayendo, pasando de una superación total en la parte más alta de la tabla, a convertirse, como ya sabemos, en el parámetro menos superado en términos globales. Por el contrario, tanto motivación como diacronía presentaban un ascenso constante a lo largo de toda la progresión, aunque mucho más acusado en el caso de la diacronía.

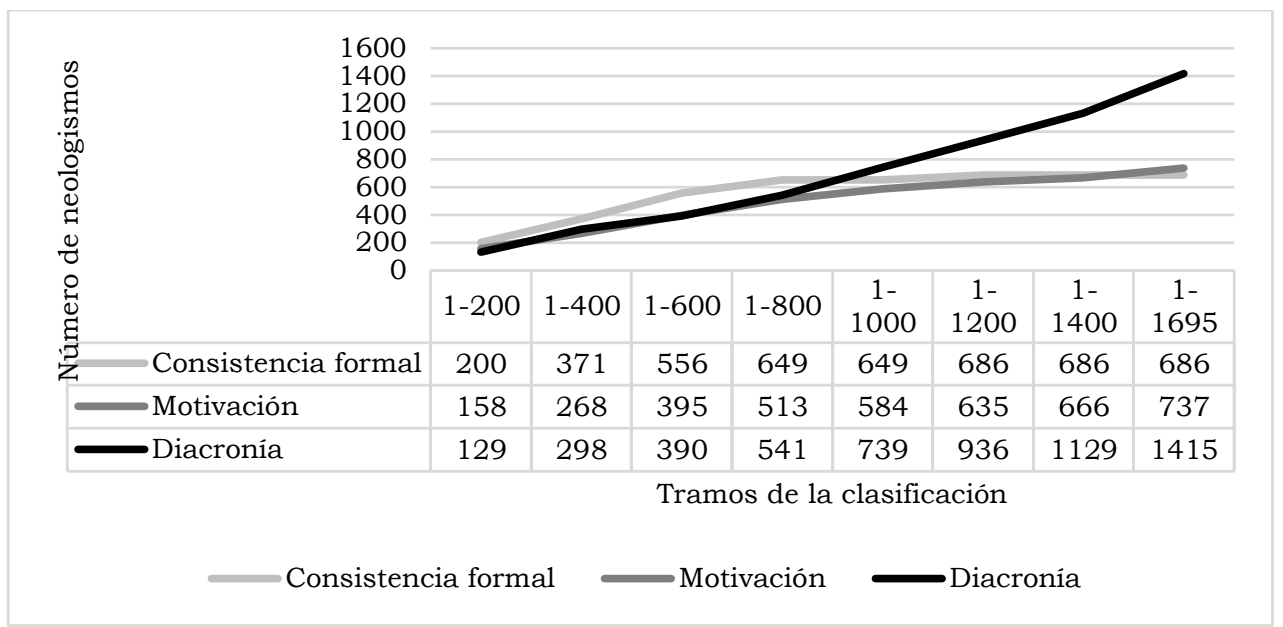

Gráfico 3: Número de neologismos que, habiendo superado un parámetro, superan los otros dos, divididos por tramos progresivos de la clasificación del baremo

En consecuencia, nos topamos con un dato paradójico: la consistencia formal era el parámetro menos superado por nuestros neologismos, como ya constatamos en la Tabla 3, pero a la vez, el más superado por aquellos con mayor puntuación. Y sabemos, por los datos obtenidos al hallar a correlación global, que una alta puntuación en diacronía suele implicar una baja puntuación en consistencia formal, así que debemos apuntar a la motivación como parámetro que favorece la consistencia formal. Dicho de otra 
manera: en nuestro estudio, los neologismos tendian a estar motivados y a ser consistentes formalmente de una manera bastante pareja.

En segundo lugar, emparejamos los tres parámetros, como muestra la Figura 2. Organizamos la clasificación en función de los puntos obtenidos por las unidades en uno de los dos parámetros del par (parámetro A). Ya no se trataba de ordenar los 1695 préstamos de acuerdo con su puntación global y ver cuántos superaban cada parámetro, sino de reordenarlos en función de la puntuación obtenida en un parámetro en concreto.

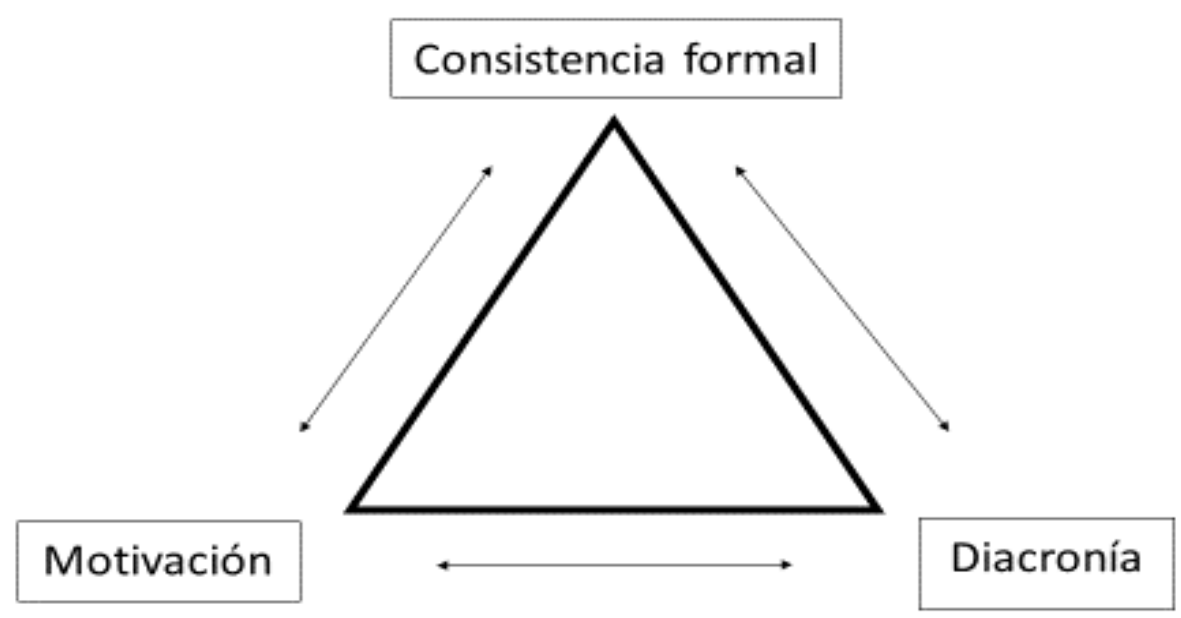

Figura 2: Emparejamiento de parámetros

En tercer lugar, comparamos el número de unidades que superaban el otro parámetro (parámetro B), y observamos en qué lugar estaban en la clasificación que acabábamos de realizar. Esta comparación no la hicimos de un modo global (eso ya lo habíamos hecho, tal y como refleja la Tabla 3) sino, de nuevo, de manera progresiva. Por último, realizamos el segundo y tercer paso de nuevo, con los mismos parámetros, pero a la inversa: el parámetro de contraste (B) pasó a ser el de referencia (A), y viceversa.

A continuación presentamos la información recabada siguiendo este método. Con el fin de completar los datos de cada par, hemos añadido la distancia media entre vectores, en número de neologismos, de cada uno de los pares analizados:

Par 1. Consistencia formal y Motivación 


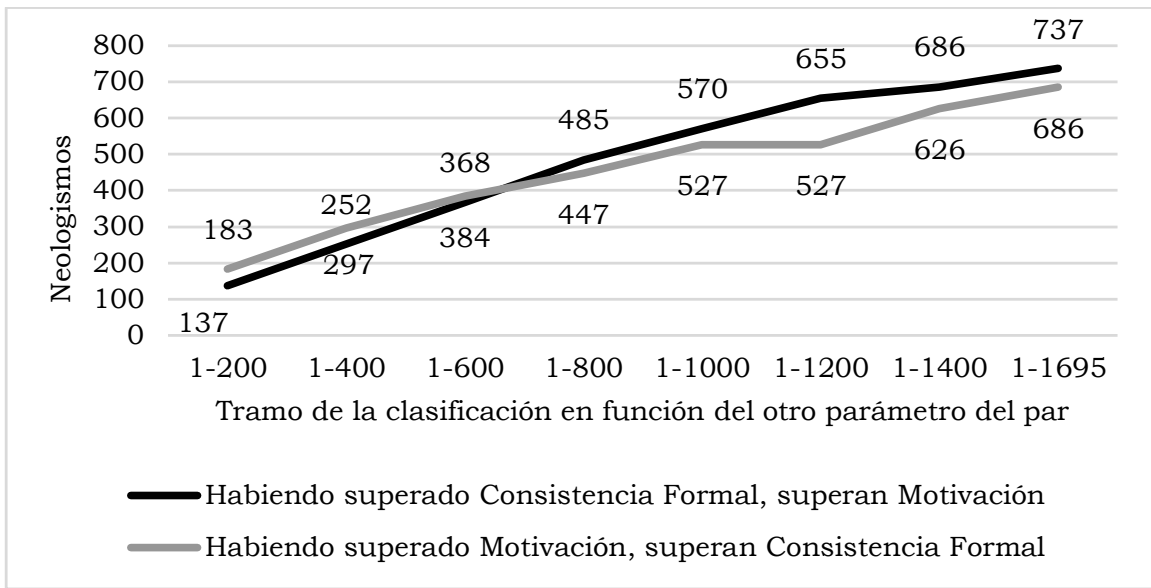

Gráfico 4: Número de neologismos que, habiendo superado un parámetro, superan los otros dos, divididos por tramos progresivos de la clasificación del baremo (par Consistencia formal - Motivación)

\section{Par 2. Consistencia formal y Diacronía}

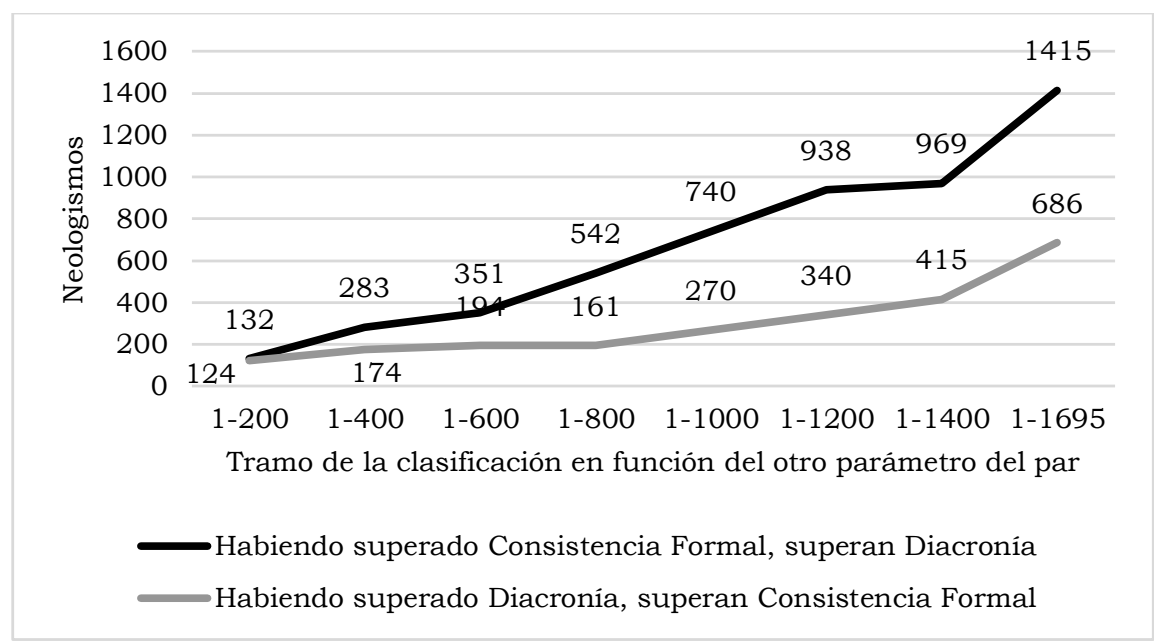

Gráfico 5: Número de neologismos que, habiendo superado un parámetro, superan los otros dos, divididos por tramos progresivos de la clasificación del baremo (par Consistencia formal - Diacronía)

\section{Par 3: Motivación y Diacronía}




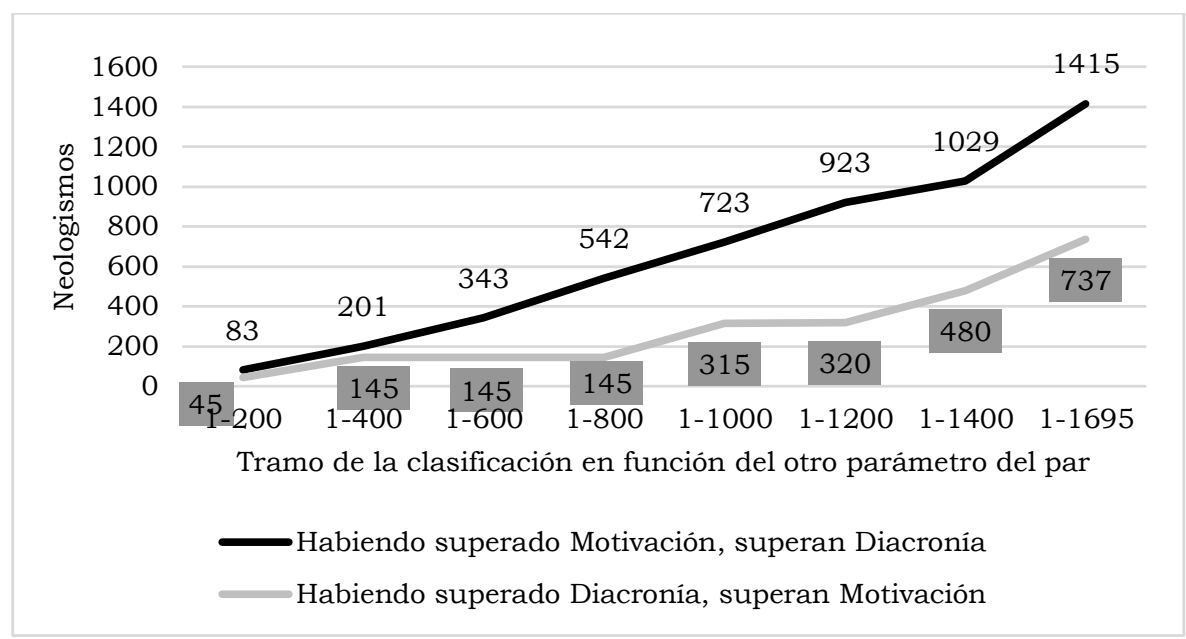

Gráfico 6: Número de neologismos que, habiendo superado un parámetro, superan los otros dos, divididos por tramos progresivos de la clasificación del baremo

(par Motivación - Diacronía)

\section{RESUltados Y Discusión}

Los datos representados en estos gráficos y tablas nos permitieron constatar varios hechos:

Divergencia media entre vectores: este dato se extrae con facilidad de las gráficas y resulta especialmente revelador. Con él pretendiamos calibrar la distancia media que existía entre los parámetros, en número de neologismos, y de acuerdo con nuestra clasificación y nuestro contraste, ya que consideramos que también podía ser un indicador de la correlación de los parámetros analizados.

\begin{tabular}{|c|c|c|c|}
\hline & Motivación & $\begin{array}{c}\text { Consistencia } \\
\text { formal }\end{array}$ & Diacronia \\
\hline Motivación & - & 53,375 & 365,875 \\
\hline Consistencia formal & 53,375 & - & 371,625 \\
\hline Diacronía & 365,875 & 371,625 & - \\
\hline
\end{tabular}

Tabla 5: Divergencia media entre vectores (en número de neologismos)

Observamos que, mientras el número medio de neologismos que separaban a la motivación de la consistencia formal a medida que ampliábamos el tramo de clasificación contrastada era de 53,375, este ascendía a 371,625 en el caso del contraste entre diacronía y consistencia formal y a 365,875 cuando comparábamos Diacronía y Motivación. El hecho de que esta desviación aumentase cuando la diacronía entraba en liza nos hizo pensar, de nuevo, en el peso específico de este parámetro en la caracterización de las 
unidades estudiadas, que solo podía verse compensada por la combinación de los otros dos parámetros, nunca por cada uno de ellos por separado.

Relación entre parámetros: las líneas de vectores que muestran los gráfi$\cos 4,5$ y 6 nos permitieron corroborar que la consistencia formal estaba subordinada en las dos parejas en las que aparecía, mientras que la diacronía se imponía en las dos suyas y, por último, que la motivación se imponía a la consistencia formal, pero perdia frente a la diacronía. Esto, unido al coeficiente de correlación de Pearson, que marcaba una significativa divergencia entre diacronia y consistencia formal y a la divergencia media entre vectores que acabamos de explicar, nos hizo concluir que las correlaciones existentes entre nuestros tres parámetros podrian ser las representadas en la Figura 3:

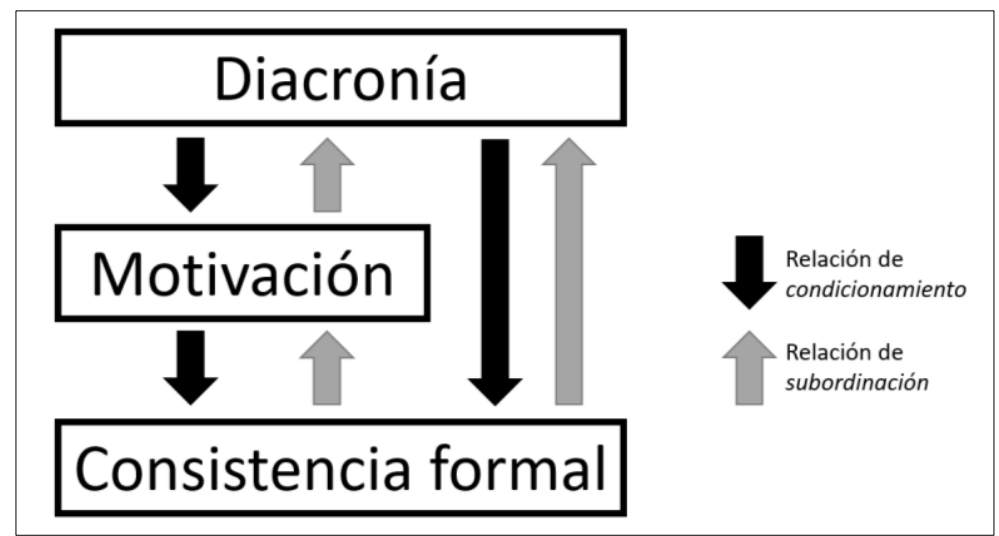

Figura 3: Relaciones de subordinación y condicionamiento entre parámetros

No podemos perder de vista al interpretar los datos y los resultados obtenidos, así como la correlación que deducimos de los tres parámetros estudiados, que todo descansa sobre unas premisas coherentes y sistemáticas, pero no por ello menos circunstanciales ni arbitrarias. Por otra parte, existen otros aspectos ligados a los neologismos que podrían complementar la metodología que planteamos. El baremo de puntuación podría haberse refinado aún más, teniendo en cuenta muchos otros factores, como las variantes formales recomendadas por la norma, el contraste con corpus mucho más actualizados, o análisis del uso de marcas tipográficas, por mencionar tan solo algunos ejemplos.

A pesar de estas limitaciones, hemos podido extraer un grupo de claves reseñables que podrían apuntar a nuevos enfoques en la caracterización de los neologismos: resulta llamativa, por ejemplo, la divergencia en el peso de los parámetros a la hora de caracterizar a los neologismos formados por sufijación frente a los prefijados. También es destacable el caso de la composición: hemos constatado que la culta es mucho menos transparente y estable que la patrimonial, algo que pone en entredicho lo afirmado por muchos autores (García Palacios, 2001; Varela Ortega, 2005; Casado Velarde, 
2015 o Maroto y Sánchez Ibáñez, 2016) y justifica la pertinencia de dividir el análisis de estos compuestos, a pesar de que muchas veces la línea que los divide sea difusa. Por último, hemos observado el peso apabullante de la dimensión diacrónica en la caracterización de las abreviaciones y acrónimos neológicos, muy por encima de su hipotética motivación o consistencia formal.

\section{Conclusiones}

A la luz de nuestros datos, podemos concluir que existe una relación directa entre los tres parámetros analizados, que puede evidenciarse mediante métodos cuantitativos y estadísticos. A pesar de las múltiples maneras en que nuestro método podría completarse, creemos que los resultados obtenidos son significativos por la coherencia interna y la sistematicidad que ha marcado todos los pasos que hemos dado, y que pueden marcar una nueva vía para el estudio cuantitativo de la neología léxica.

Todos los análisis y desgloses de correlaciones que hemos llevado acabo nos permiten apreciar la existencia de dos tipos de parámetros: por un lado, la diacronía, entendida como una combinación de implantación, uso reciente y referencia a realidades actuales, que se convierte el factor de peso, determinante en la caracterización y en la relevancia de las unidades analizadas, y por el otro la motivación y la consistencia formal, que claramente están subordinados al primer parámetro. En concreto, hemos probado que, de acuerdo con nuestros datos y análisis, el aumento del baremo de uno lleva parejo, en mayor o menor medida, el aumento del baremo de los otros dos. Se trata, pues, de una correlación directa.

En definitiva, hemos presentado un estudio muy concreto y limitado, pero fácilmente exportable a otros contextos neológicos, que puede abrir la vía a definir a los neologismos en positivo, atendiendo a lo que los caracteriza, y no reseñando aquello de lo que carecen. Podemos concluir que, de acuerdo con nuestro análisis, los neologismos unidades léxicas esencialmente recientes, y, en menor medida, motivadas y consistentes.

\section{REFERENCIAS}

BATTANER ARIAS, M. P. (dir.) (2001): Lema. Diccionario de Lengua Española VOX, Barcelona: Spes.

BOULANGER, J. C. (2010): "Sur l'existence des concepts de "néologie" et de 'néologisme' Propos sur un paradoxe lexical et historique", Actas del I Congreso Internacional de Neología en las lenguas románicas, Barcelona: Institut
Universitari de Lingüistica Aplicada, Universidad Pompeu Fabra, pp. 31-74.

CABRÉ, M. T. (1999): La terminología. Representación y comunicación. Una teoria de base comunicativa $y$ otros artículos, Barcelona: Institut Universitari de Lingüística Aplicada, Universitat Pompeu Fabra.

CABRÉ M. T. (2015): "La neologia: un nou camp a la cerca de la seva 
consolidació científica", Caplletra, 59, pp. 125-136.

CABRÉ, M. T. (2016): "Principios y parámetros en una teoría de los neologismos", García Palacios, J.; De Sterck, G.; Linder, D, Maroto, N.; Sánchez Ibáñez, M.; Torres del Rey, J. (eds.): La neología en las lenguas románicas, Frankfurt: Peter Lang, pp. 2742.

CASADO VELARDE, M. (2015): La innovación léxica en el español actual, Madrid: Síntesis.

ESTORNELL, M. (2009): El reconocimiento de neologismos y su caracterización en un corpus de prensa escrita (2004-2007), Valencia: Universidad de Valencia.

FREIXA, J. y SOLÉ, E. (2006): “Análisis lingüístico de la detección automática de neologismos léxicos", Sendebar, 17, pp. 135147.

FUNDACIÓN DEL ESPAÑOL URGENTE. www.fundeu.es

GARCÍA PALACIOS, J. (2001): "La quimera del euro-. Acerca de algunos elementos compositivos en español", Revista de Lexicografia, 7, pp. 33-62.

GUILBERT, L. (1973) : "Théorie du néologisme", Cahiers de l'Association internationale des études francaises, 25, pp. 9-29.

GUILBERT, L. (1975) : La créativité lexicale, Paris : Larousse.

HORMIGO, DÍAZ, T. M. (2007). "Aproximación lingüística a la neología léxica". Morfologia: Investigación, docencia, aplicaciones. Actas del II Encuentro de Morfologia: Investigación y Docencia, Cáceres, 5, pp. 33-54.

LLOPART-SAUMELL, E. (2016): La funció dels neologismes: revisió de la dicotomia neologisme denominatiu $i$ neologisme estilístic (Tesis doctoral no publicada), Barcelona: Universidad Pompeu Fabra.
LOUBIER, C. (2011): De l'usage de l'emprunt linguistique, Montreal: Office Québecoise de la Langue Francaise.

MAROTO, N. \& SÁNCHEZ IBÁÑEZ, M. (2016): "Viticultores, pero enoturistas: Variación neológica y registros de uso en el lenguaje del vino", Sánchez Manzanares, C. y Azorín Fernández, D. (eds.) Estudios de neología del espanol, Murcia: Editum, pp. 153177.

OBNEO (Observatori de Neologia) (2004): Metodologia del trabajo en neología: criterios, materiales $y$ procesos, Barcelona: Institut Universitari de Lingüistica Aplicada, Universitat Pompeu Fabra.

QUIRION, J. (2003): "Methodology for the design of a standard research protocol for measuring terminology usage", Terminology, 9(1), pp. 29-49.

REAL ACADEMIA ESPAÑOLA. (2014): Diccionario de la Lengua Espanola. $\left(23^{\mathrm{a}}\right.$ edición), Madrid: Espasa.

REY, A. (1976) : "Néologisme: un pseudo-concept?", Cahiers de Lexicologie: Revue Internationale de Lexicologie et Lexicographie, 28, pp. 3-17.

SABLAYROLLES，J. F. (2000) : La néologie en français contemporain. Examen $d u$ concept et analyse des productions néologiques récentes, Paris: Honoré Champion.

SABLAYROLLES, J. F. (2016): "Néologismes et idées recues", García Palacios, J.; De Sterck, G.; Linder, D.; Maroto, N.; Sánchez Ibáñez, M.; Torres del Rey, J. (eds.): La neología en las lenguas románicas, Frankfurt: Peter Lang, pp. 43-56.

SABLAYROLLES, J. F. \& THOIRON, F. (2003): "Le sentiment 
néologique", L'innovation lexicale. Paris: Honoré Champion.

SÁNCHEZ MANZANARES, C. (2013): "Valor neológico y criterios lexicográficos para la sanción y censura de neologismos en el diccionario general", Sintagma: revista de lingüistica, 25, pp. 111-125.
VARELA ORTEGA, S. 2005. Morfología léxica: la formación de palabras, Madrid: Gredos.

VEGA MORENO, E. (2015): "La creación neológica como herramienta publicitaria”, González Sanz, M. (coord.), Jóvenes aportaciones a la investigación lingüística, Sevilla: Alfar, pp. 351365.

ANEXo 1

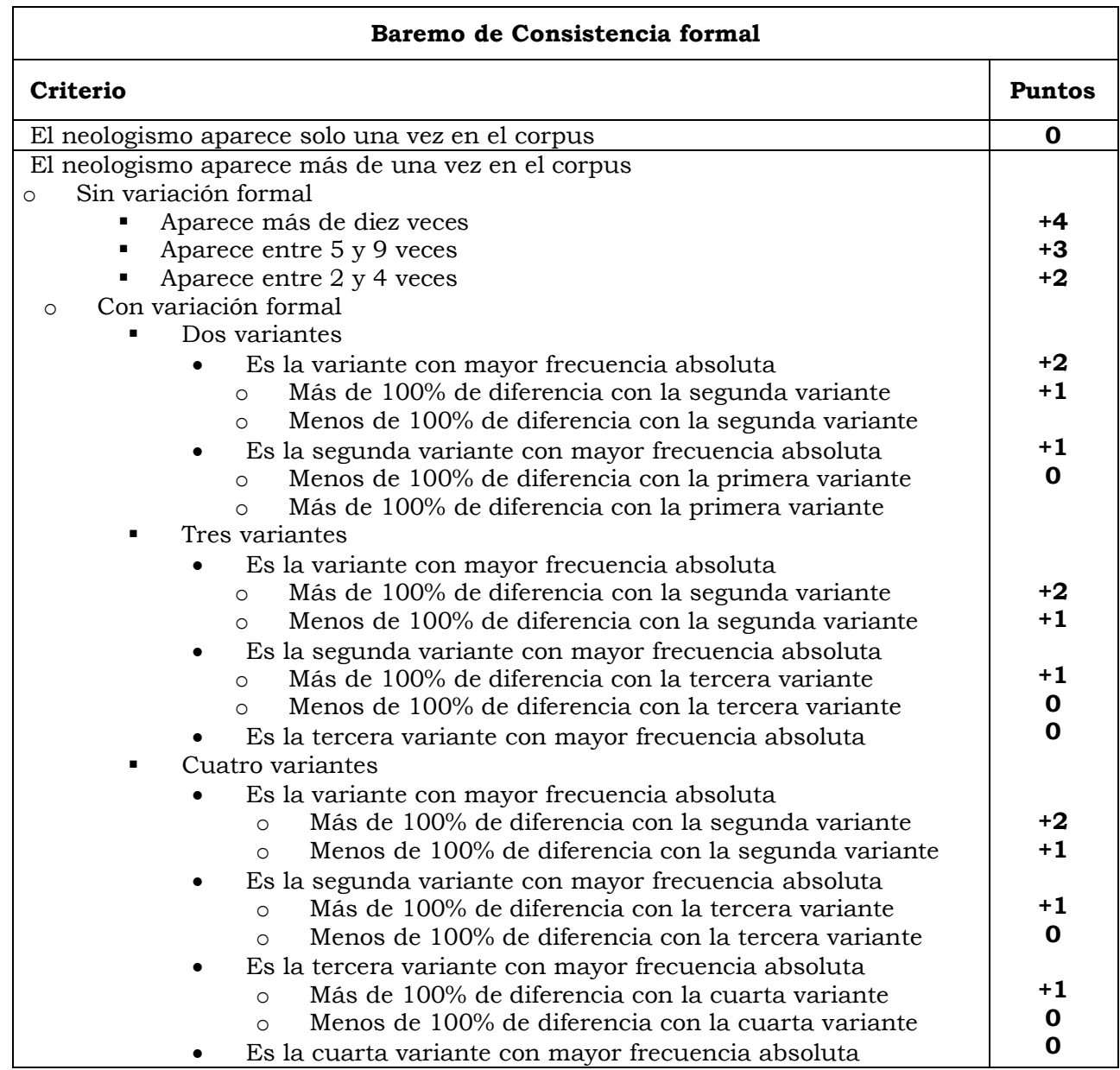




\section{Baremo de Motivación}

\begin{tabular}{|c|c|}
\hline Criterio & Puntos \\
\hline 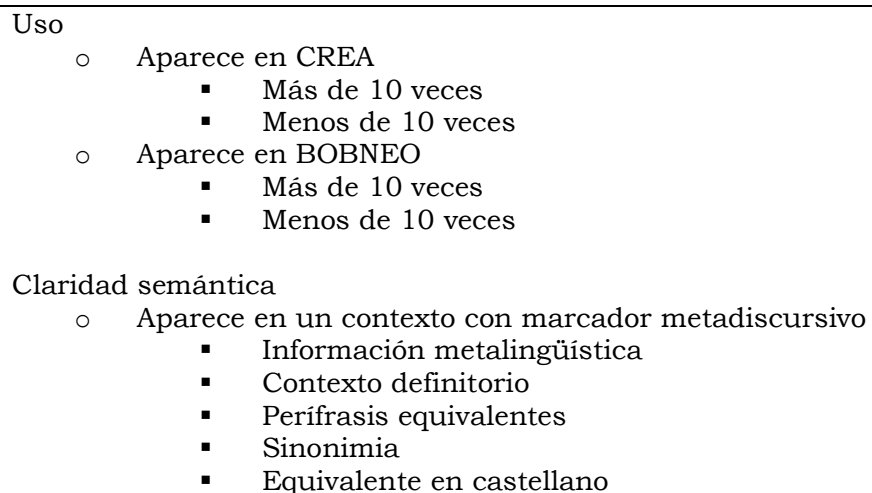 & $\begin{array}{l}+2 \\
+1 \\
+2 \\
+1\end{array}$ \\
\hline
\end{tabular}

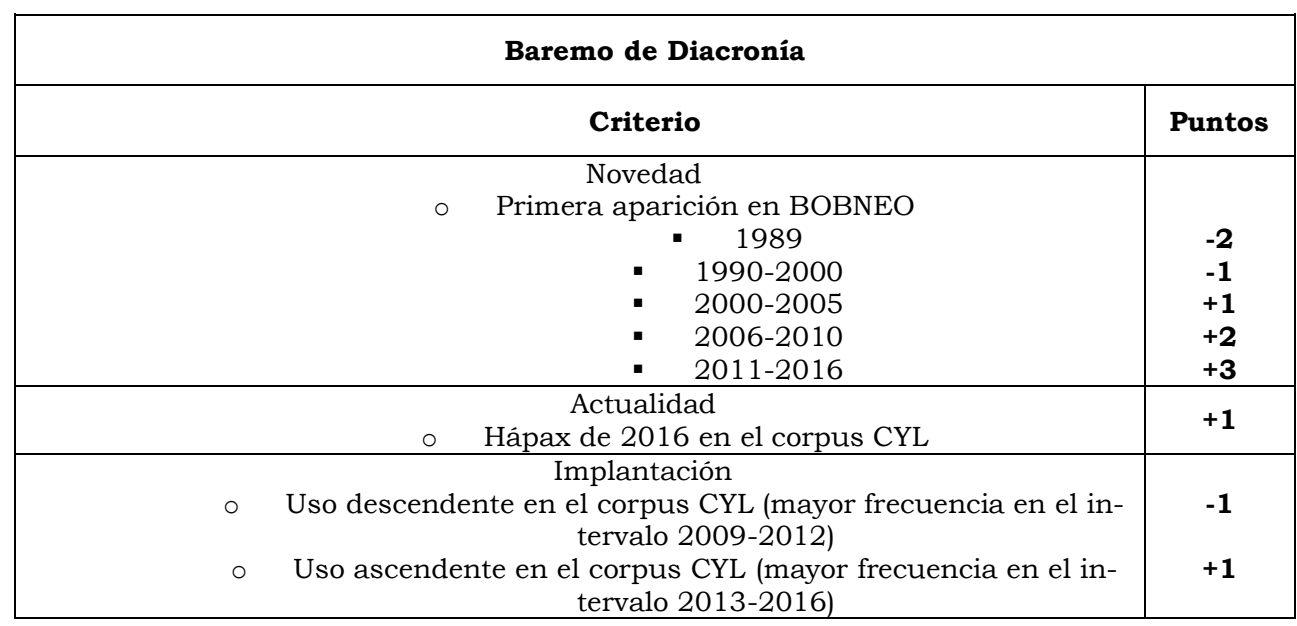


Anexo 2

\begin{tabular}{|c|c|c|c|c|c|c|c|c|c|c|c|c|c|}
\hline \multirow{3}{*}{ Neologismo } & \multirow{4}{*}{$\begin{array}{c}\text { Consisten- } \\
\text { cia formal } \\
\text { TOTAL } \\
4\end{array}$} & \multicolumn{6}{|c|}{ Motivación } & \multicolumn{5}{|c|}{ Diacronia } & \multirow{3}{*}{$\begin{array}{c}\text { Puntuación } \\
\text { global }\end{array}$} \\
\hline & & \multirow{3}{*}{$\begin{array}{c}\text { Puntos } \\
\text { marca- } \\
\text { dores } \\
\text { meta- } \\
\text { disc. }\end{array}$} & \multicolumn{4}{|c|}{ Uso } & \multirow{3}{*}{$\begin{array}{c}\text { TOTAL } \\
10\end{array}$} & \multirow{2}{*}{\multicolumn{2}{|c|}{$\begin{array}{c}\text { Novedad } \\
\text { Año } 1^{\text {a }} \text { apari- } \\
\text { ción BOB- } \\
\text { NEO } \\
\end{array}$}} & \multirow{3}{*}{$\begin{array}{c}\begin{array}{c}\text { Implanta- } \\
\text { ción }\end{array} \\
\begin{array}{c}\text { Puntos } \\
\text { impl. (CYL) }\end{array} \\
1\end{array}$} & \multirow{3}{*}{$\begin{array}{c}\begin{array}{c}\text { Actuali- } \\
\text { dad }\end{array} \\
\text { Puntos } \\
\text { hápax } \\
2016 \\
\end{array}$} & \multirow{3}{*}{$\begin{array}{c}\text { TOTAL } \\
\\
4 \\
\end{array}$} & \\
\hline & & & \multicolumn{2}{|c|}{ CREA } & \multicolumn{2}{|c|}{$\begin{array}{l}\text { BOB- } \\
\text { NEO }\end{array}$} & & & & & & & \\
\hline fracking & & & & 0 & 84 & 2 & & 2011 & 3 & & & & 18 \\
\hline doodle & 4 & 4 & 7 & 1 & 109 & 2 & 7 & 2010 & 3 & 1 & & 4 & 15 \\
\hline$a p p$ & 4 & 2 & 10 & 1 & 375 & 2 & 5 & 2010 & 3 & 1 & & 4 & 13 \\
\hline like & 3 & 2 & 103 & 2 & 31 & 2 & 6 & 2011 & 3 & 1 & & 4 & 13 \\
\hline micromecenazgo & 3 & 4 & & 0 & 31 & 2 & 6 & 2011 & 3 & 1 & & 4 & 13 \\
\hline coworking & 2 & 4 & & 0 & 18 & 2 & 6 & 2011 & 3 & 1 & & 4 & 12 \\
\hline benzodiacepina & 2 & 2 & 15 & 2 & 3 & 1 & 5 & 2011 & 3 & 1 & & 4 & 11 \\
\hline bumping & 2 & 4 & & 0 & 3 & 1 & 5 & 2013 & 3 & 1 & & 4 & 11 \\
\hline ceas & 2 & 4 & 1 & 1 & & 0 & 5 & 2014 & 3 & 1 & & 4 & 11 \\
\hline enoturismo & 4 & 2 & & 0 & 60 & 2 & 4 & 2008 & 2 & 1 & & 3 & 11 \\
\hline mindfulness & 2 & 4 & & 0 & 9 & 1 & 5 & 2012 & 3 & 1 & & 4 & 11 \\
\hline nitrometano & 2 & 6 & 2 & 1 & 9 & 1 & 8 & 2007 & 2 & -1 & & 1 & 11 \\
\hline sociosanitario -ria & 4 & & 75 & 2 & 112 & 2 & 4 & 2006 & 2 & 1 & & 3 & 11 \\
\hline troquelador -ora & 2 & 2 & 6 & 1 & 14 & 2 & 5 & 2014 & 3 & 1 & & 4 & 11 \\
\hline alistano -na & 4 & & 55 & 2 & & 0 & 2 & 2011 & 3 & 1 & & 4 & 10 \\
\hline combinativo -va & 3 & & 6 & 1 & 41 & 2 & 3 & 2010 & 3 & 1 & & 4 & 10 \\
\hline desarrollador-ora & 4 & & 143 & 2 & 331 & 2 & 4 & 2002 & 1 & 1 & & 2 & 10 \\
\hline desprivatizar & 2 & 2 & 7 & 1 & 3 & 1 & 4 & 2013 & 3 & 1 & & 4 & 10 \\
\hline due diligence & 2 & 4 & 2 & 1 & 15 & 2 & 7 & 2007 & 2 & -1 & & 1 & 10 \\
\hline geoestratégico -ca & 3 & & 62 & 2 & 106 & 2 & 4 & 2009 & 2 & 1 & & 3 & 10 \\
\hline
\end{tabular}

